\title{
Oral UFT (uracil plus futrafur) for neoadjuvant chemotherapy of gastric cancer
}

\author{
Yoshinori Nio, Chikage Iguchi, Kunihiro Yamasawa, Masayuki Itakura, Hiroshi Omori, \\ Koji Hashimoto, Seiji Yano, Shoichiro Sumi, and Katsuhiro Tamura
}

First Department of Surgery, Shimane Medical University, 89-1, Enya-cho, Izumo Shimane 693-8501, Japan

\begin{abstract}
:
Background. Neoadjuvant chemotherapy has become one of the topics of interest in chemotherapy of gastric cancer; the present study assessed the clinical benefits of neoadjuvant chemotherapy with oral uracil and futrafur (UFT) for gastric cancer.

Methods. Between 1991 and 1997, 82 patients with gastric cancer (36 with early and 46 with advanced cancers) received UFT at $300-600 \mathrm{mg} /$ day orally for $1-6$ weeks before surgery. Objective responses, histological effects, and postsurgical survival rates were assessed.

Results. In 69 of the 82 patients, the objective responses of the primary lesions were assessed by endoscopy or upper gastrointestinal series examination, and 2 complete responses (CR)s, 25 partial responses (PRs), and 42 no changes (NCs) were seen (39.1\% response). Histological effects were evaluated in 82 patients, and 2 grade 3,11 grade 2,11 grade $1 \mathrm{~b}, 27$ grade 1a, and 31 grade 0 effects were seen. A longer period of UFT administration was associated with a CR or PR. However, the objective responses did not correlate with the histological effects. All the patients underwent gastrectomy, and during the median follow-up period of 41 months, 3-year survival rates were $97.1 \%$ for pTNM stage $1,75 \%$ for stage 2 , $86.7 \%$ for stage 3 , and $41.6 \%$ for stage 4 . The survival rates of stage 3 and stage 4 patients were higher than those of the historical controls in our department. However, CR or PR did not correlate with the improvement in survival. Side effects before surgery were not serious; they included slight myelotoxicity, liver dysfunction, and anorexia; however, 3 patients $(3.7 \%)$ had suture insufficiency, 3 patients $(3.7 \%)$ had methicillin-resistant Staphylococcus aureus (MRSA) enteritis, and 7 patients $(8.5 \%)$ had liver dysfunction.

Conclusions. Preoperative chemotherapy for gastric cancer with oral UFT was safe and resulted in a good local response (macro- and microscopically) which may indicate the possibility of improved survival with neoadjuvant chemotherapy with UFT. Furthermore, preoperative chemotherapy with oral UFT is easy and patients can receive this treatment on an outpatient basis.
\end{abstract}

Offprint requests to: $\mathrm{Y}$. Nio

Received for publication on Feb. 5, 1999; accepted on March 15,1999
Key words: gastric cancer, neoadjuvant chemotherapy, UFT

\section{Introduction}

Although the results of treatment for gastric cancer in Japan have been improving over the past two decades, gastric cancer is still one of the leading causes of cancer death in Japan. The 5-year survival rates of gastric cancer patients after gastrectomy have reached more than $90 \%$ for stage 1 and $70 \%-80 \%$ for stage 2 . However, the prognosis of patients with stage 3 or 4 gastric cancer is still poor, and 5-year survival rates are about $40 \%$ for stage 3 and $5 \%-10 \%$ for stage 4 . In order to improve the treatment results for gastric cancer, a variety of therapies have been employed, and chemotherapy has played the most important role. However, it is still unclear whether postoperative adjuvant chemotherapy improves survival, although gastric cancer is now considered to be relatively sensitive to chemotherapy compared with cancers of other digestive organs.

Since the introduction of cisplatin (CDDP), a variety of combination chemotherapies, including etoposide + 5-fluorouracil (5FU) + CDDP (EAP) and 5FU + Adriamycin + CDDP (FAP) regimens have been employed for the treatment of gastric cancer [1-4]. After the report by Wilke and colleagues (5), neoadjuvant chemotherapy has become one of the topics of interest in chemotherapy of gastric cancer [5-7]. The first reports on neoadjuvant chemotherapy of gastric cancer were published in Japan, in the 1960s [8,9], and since these reports, many Japanese researchers have employed various neoadjuvant chemotherapies [10-17]. The major purpose of neoadjuvant chemotherapy is preoperative downstaging, to enable more curative surgery. Previous neoadjuvant chemotherapies employed intensive chemotherapies such as EAP or FAP, and we 
have also employed an intensive 5FU + CDDP + 4'epirubicin (FPEPIR) regimen (a modified version of the FAP regimen) [18,19]; however, these therapies frequently cause serious side effects and result in the interruption or postponement of surgery; their true effects are still unclear.

In Japan, neoadjuvant chemotherapy regimens have been different from those used in Western countries, because many neoadjuvant regimens have included oral 5-FU or a mixture of uracil and futrafur at 4:1 (UFT) $[12,13,16,17]$. In general, the choice of adjuvant chemotherapy for gastric cancer in Japan has differed from that in the United States and European countries; in Japan, oral chemotherapy with fluoropyrimidines has been the standard regimen for gastric cancer. Among these oral fluoropyrimidines, UFT is the most popular agent in Japan. The side effects of oral UFT are not serious; accordingly, UFT is used especially for adjuvant chemotherapy after surgery on an outpatient basis in Japan [20-22]. The response rates of digestive organ cancers to UFT alone in Japan were reported to be: gastric cancer, $27.7 \%$; colorectal cancer, $25.0 \%$; liver cancer, $19.2 \%$; pancreatic cancer, $25.0 \%$; and gallbladder or bile duct cancer, $25.0 \%$ [23]. These results are similar to those of intensive intravenous chemotherapies. Accordingly, UFT can be employed for neoadjuvant chemotherapy instead of these intensive chemotherapies, and neoadjuvant chemotherapy with oral UFT may have a major advantage, in that UFT can be administered on an outpatient basis. To achieve downstaging during the waiting period for surgery, we have employed preoperative chemotherapy with oral UFT from 1991, and a total of 82 patients have received this therapy. In the present study, the clinical benefits of preoperative chemotherapy with oral UFT for gastric cancer during the waiting period for surgery were assessed with regard to the objective response and the benefits in terms of postsurgical survival.

\section{Patients and methods}

\section{Patients}

Two basic criteria for preoperative chemotherapy with UFT had to be met before administration: (1) histological or cytological proof of gastric cancer, and (2) performance status $\leq 3$ on the European Cooperative Oncology Group (ECOG) scale. Contraindications included: (1) total disability (performance status $[\mathrm{PS}]=4$, ECOG score), (2) prior chemotherapy, radiotherapy, or immunotherapy within 4 weeks, (3) active infectious disease, (4) severe anemia (hemoglobin $<9.0 \mathrm{~g} / \mathrm{dl}$ ), leukopenia $\left(<3000\right.$ white blood cells $\left./ \mathrm{mm}^{3}\right)$, thrombocytopenia $\left(<70000\right.$ platelets $\left./ \mathrm{mm}^{3}\right)$, azotemia (creati-
Table 1. Patient profile

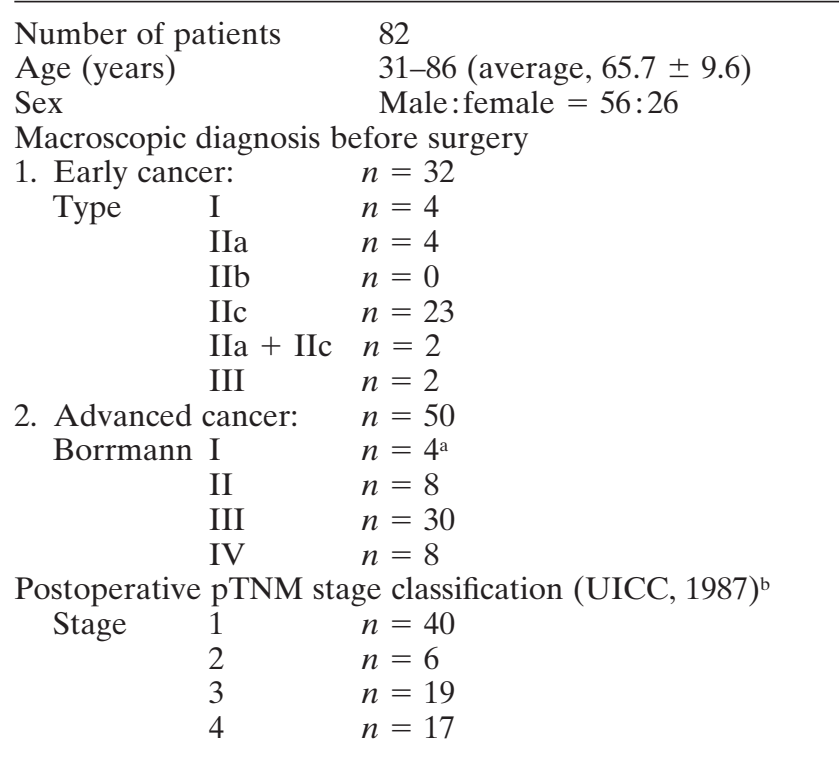

a Including one recurrent cancer of the remnant stomach

${ }^{\mathrm{b}}$ Excluding one recurrent cancer of the remnant stomach

nine $>2.0 \mathrm{mg} / \mathrm{dl}$ ), or liver dysfunction (GOT, GPT, and alkaline phosphatase $>$ four fold normal limits), (5) severe heart disease or concomitant malignant disease, and (6) pregnancy. All patients and their families were fully informed with regard to the treatment program, and informed consent was obtained.

Between 1991 and 1997, a total of 82 patients with gastric cancer were included in the present study: 36 with early (pT1) gastric cancers and 46 with (pT2-4) advanced gastric cancer according to the postoperative International Union against Cancer classification. After surgery, the stage of gastric cancer was classified according to the (UICC) TNM stage classification (1987). The patient profile is summarized in Table 1.

\section{Treatment protocol for neoadjuvant chemotherapy with UFT}

The patients were administered UFT orally after meals during the waiting period for surgery. The administration usually started from the first visiting day to the outpatient ward and the patients were given the last UFT at 6 p.m. on the day before surgery. One capsule of UFT includes $100 \mathrm{mg}$ of futrafur (FT), and the dose of UFT is usually expressed as the does of FT. Three different doses $(300,400$, and $600 \mathrm{mg} /$ day) were used, and the dose of UFT for each patient was determined according to the patients condition (PS, body weight, age, hematology, and serum biochemistry), because UFT sometimes causes serious myelotoxicity or hepatotoxicity. 
Examinations of hematology, serum biochemistry, and serum tumor markers, and evaluations of the symptomatic status and performance status were routinely performed at weekly or biweekly intervals, sometimes more frequently. The size of the primary lesion was usually assessed before UFT administration and 1 or 2 days before surgery by endoscopy and/or upper gastrointestinal series examination, sometimes more frequently. If the disease appeared to progress or serious side effects were seen, the patients were either managed symptomatically and supportively or offered alternative experimental regimens if their general condition seemed to be appropriate.

\section{Postsurgical adjuvant chemotherapy}

The patients were treated with adjuvant chemotherapy according to their postsurgical stage classification. Stage 1-3 patients received adjuvant chemotherapy with UFT for 1-3 years and stage 4 patients received intensive chemotherapy with one to four courses of CDDP, 5-FU, and epirubicin (FPEPIR regimen) [19] according to their condition and then received oral UFT daily for as long as possible.

\section{Evaluation of objective response and histopathological effects}

The objective response was evaluated as complete response (CR), partial response (PR), no change (NC), or progressive disease (PD) by endoscopy, upper gastrointestinal series examination, and computed tomography (CT) scan, and the histopathological effect was evaluated based on the grade (0-3), according to the criteria of the Japanese Research Society for Gastric Cancer (1995, First English Edition). The duration of the response was not included in the evaluation of the objective response, because all patients underwent surgery.

\section{Evaluation of side effects}

The WHO standard criteria for toxicity [24] were used.

\section{Follow-up of patients}

All patients were followed-up by physical examination, general X-ray examination, ultrasonography (US), CT, routine hematologic and biochemical examinations, and serum tumor marker assays.

\section{Assay of drug concentrations}

The concentration of FT was measured by highpressure liquid chromatography, and that of 5-FU was measured by gas-mass chromatography according to the method described by Marunaka et al. [25].

\section{Statistical evaluation}

The response rate of the primary lesion, the histological effects, and the survival rate after surgery were evaluated to judge the effects of the therapies. $\chi^{2}$ and Mann-Whittney $U$-tests were used to compare patient backgrounds among the three dosage groups. Overall survival was calculated by the Kaplan-Meier method. A $P$ value of less than 0.05 was considered to be significant. Statistical analysis was carried out using SAS computer software SAS Institute Inc., Cary, NC, USA.

\section{Results}

Dose and period of drug administration (Table 2)

UFT was administered at three doses $(300,400$, and $600 \mathrm{mg} /$ body per day). The administration period ranged

Table 2. Preoperative dose of UFT

\begin{tabular}{llccc}
\hline & & $\begin{array}{c}\text { Early cancer }^{\mathrm{a}} \\
(\mathrm{pT} 1, n=36)\end{array}$ & $\begin{array}{c}\text { Advanced cancer }^{\mathrm{a}} \\
(\mathrm{pT} 2-4, n=46)\end{array}$ & $\begin{array}{c}\text { Overall } \\
(n=82)\end{array}$ \\
\hline Dose (mg/body per day) & 300 & 15 & 23 & 38 \\
& 400 & 17 & 13 & 30 \\
Period (days) & 600 & 4 & 10 & 14 \\
& $\leq 14$ & 11 & 12 & 23 \\
Total dose (g) & $15-28$ & 18 & 19 & 47 \\
& $\geq 29$ & 7 & 5 & 12 \\
& $\leq 6.0$ & 14 & 18 & 32 \\
& $7-12.0$ & 20 & 23 & 43 \\
\hline
\end{tabular}

UFT, Uracil plus futrafur $4: 1$

a According to postoperative pathology 
between 7 and 40 days, and the total dose ranged between 2.4 and $21.6 \mathrm{~g}$.

\section{Side effects (Table 3)}

Side effects were not serious before surgery: anorexia in 11 patients $(13.4 \%)$, leukopenia in 3 patients $(3.7 \%)$, thrombocytopenia in 2 patients $(2.4 \%)$, and slight liver dysfunction in 2 patients $(2.4 \%)$. Postoperative complications were seen in 13 patients: 3 patients $(3.7 \%)$ with

Table 3. Side effects (evaluated in 82 patients)

\begin{tabular}{ll}
\hline I. Preoperative side effects & \\
Anorexia & $11(13.4 \%)$ \\
Massive bleeding from gastric cancer & $2(2.4 \%)$ \\
Leukopenia $\left(<300 \mathrm{WBC} / \mathrm{mm}^{3}\right.$ & $3(3.7 \%)$ \\
Thrombocytoperia $\left(<100000\right.$ platelets $\left./ \mathrm{mm}^{3}\right)$ & $2(2.4 \%)$ \\
GOT $\uparrow(>100 \mathrm{IU} / \mathrm{l})$ & $2(2.4 \%)$ \\
II. Postoperative complications & \\
Suture insufficiency & $3(3.7 \%)$ \\
MRSA enteritis & $3(3.7 \%)^{\mathrm{a}}$ \\
GOT $\uparrow(>100 \mathrm{IU} / \mathrm{l})$ & $7(8.5 \%)^{\mathrm{b}}$ \\
\hline
\end{tabular}

MRSA, Methicillin-resistant Staphylococcus aureus

${ }^{a}$ One patient had panperitonitis due to colonic perforation and two patients had enteritis

${ }^{\mathrm{b}}$ Two patients had high-level elevation of GOT, of more than $500 \mathrm{IU} / 1$ suture insufficiency, 1 with perforative peritonitis, and 2 with enteritis due to methicillin-resistant staphylococcus aureus (MRSA); all of these patients were cured by conservative therapy or re-operation. Liver dysfunction (serum GOT level > 100 IU/l) was seen in 7 patients $(8.5 \%)$ and serum GOT levels increased to more than $500 \mathrm{IU} / 1$ in 2 patients. It seemed that patients who received UFT for a long period suffered more often from postoperative complications.

\section{Objective and histological responses}

In 69 of the 82 patients, the sizes of primary tumors before and after UFT administration were compared by endoscopy or upper gastrointestinal series examination, and 2 CRs, 25 PRs, and 42 NCs were seen $(27 / 69,39.1 \%$ response). The involved nodes became smaller in 2 patients, and a single lesion of liver metastasis disappeared in 1 patient. Findings for 4 representative patients who responded to the neoadjuvant UFT treatment are shown in Figs. 1, 2. There was no relationship between the response and the clinical stage (Table 4). Table 5 summarizes the relationship between the response and the dose and administration period. The results also suggest that the dose may not be a very important factor, but longer periods of UFT administration, espe-
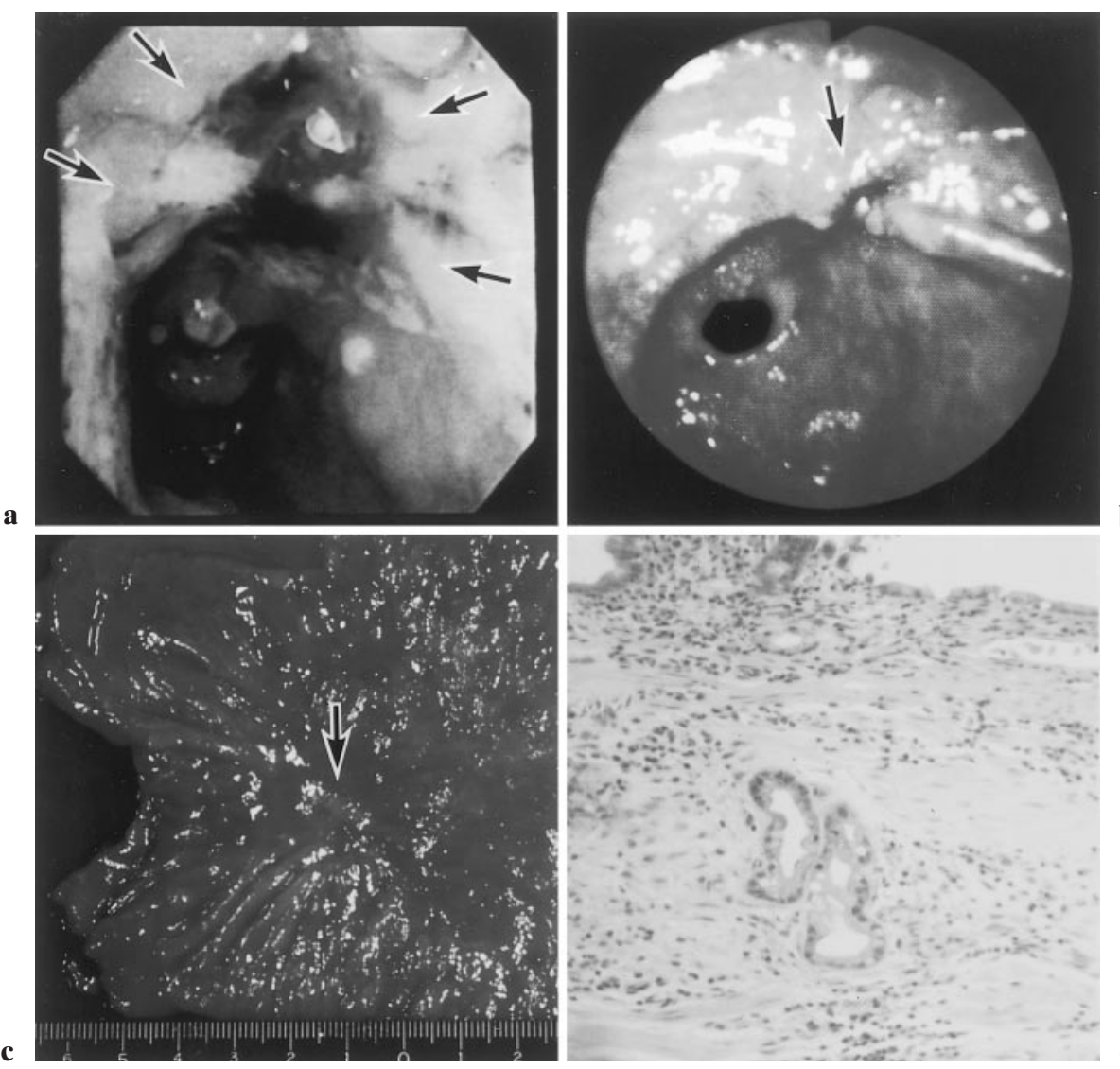

Fig. 1a-d. Representative patient with complete response (CR). The patient, a 70-year-old woman, received uracil + futrafur, 4:1 (UFT) at $300 \mathrm{mg} /$ day for 16 days. a Before UFT: IIc-like advanced gastric cancer at lesser curvature of the prepyloric region. b After UFT: scar formation of ulcerative lesion; objective response was evaluated as CR. c Resected specimen: no macreoscopic lesion was seen. d Pathology: minimal lesion of well differentiated adenocarcinoma; the histological response was evaluated as grade 2 . The postoperative stage classification was stage 1a: pT1 (m), pN0, M0 
Table 4. Macroscopic and histological response after preoperative UFT

\begin{tabular}{lccc}
\hline & \multicolumn{2}{c}{ Postoperative stage $^{\mathrm{a}}$} & \\
\cline { 2 - 3 } Effect & Stage $(1 n=40)$ & Stage 2-4 $(n=42)$ & Overall $(n=82)$ \\
\hline Macroscopic response & $2(5.6 \%)$ & 0 & $2(2.9 \%)$ \\
CR & $14(38.9 \%)$ & $11(33.3 \%)$ & $25(36.2 \%)$ \\
PR & $20(55.5 \%)$ & $22(66.7 \%)$ & $42(60.9 \%)$ \\
NC & 0 & 0 & 0 \\
PD & 4 & 9 & 13 \\
Unevaluable & $1(2.5 \%)$ & $1(2.4 \%)$ & \\
Histological effect & $5(12.5 \%)$ & $6(14.3 \%)$ & $2(2.5 \%)$ \\
Grade 3 & $3(7.5 \%)$ & $8(19.0 \%)$ & $11(13.4 \%)$ \\
Grade 2 & $13(32.5 \%)$ & $14(33.3 \%)$ & $27(32.9 \%)$ \\
Grade 1b & $18(45.0 \%)$ & $13(31.0 \%)$ & $31(37.8 \%)$ \\
Grade 1a & & & \\
Grade 0 & & & \\
CR Com $)$ &
\end{tabular}

CR, Complete response; PR, partial response; NC, no change; $\mathrm{PD}$, progressive disease ${ }^{a}$ pTNM, (UICC 1987)

Table 5. Administered dose and macroscopic response after preoperative UFT (evaluated in 69 of 82 patients)

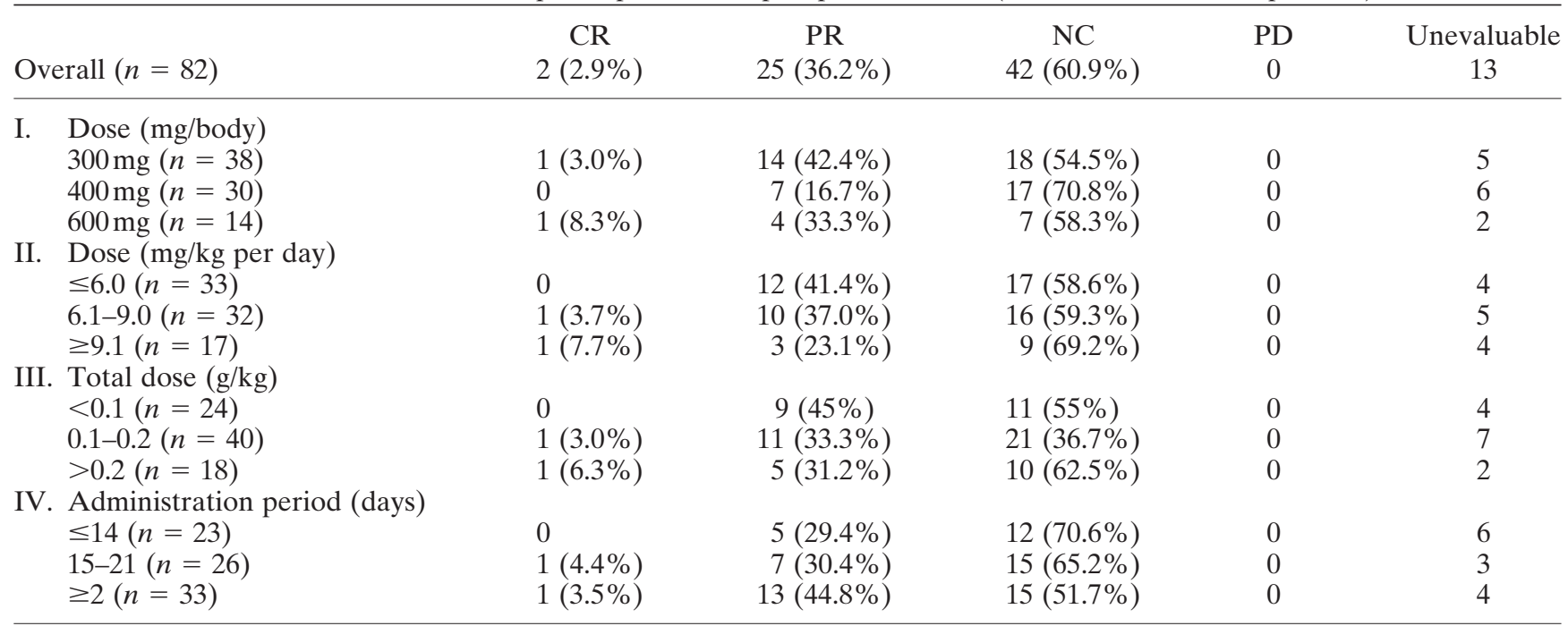

cially for more than 3 weeks, may be associated with good objective responses.

The histological effect was evaluated in 82 patients and the following classifications were made: grade 3 (complete disappearance or necrosis of tumor cells), 2; grade 2 (necrotic changes $>2 / 3$ area), 11; grade $1 b(>1 /$ 3 area), 11; grade $1 \mathrm{a}$ ( $<1 / 3$ area), 27; and grade 0 (no histological changes), 31 (Table 4). There was a correlation between the histological effect and depth of the primary tumor, and the frequences of grade 2-3 responses were significantly higher $(P<0.05)$ in pT4 tumors than in pT1-3 tumors (Table 4$)$. There was no relationship between the histological response and the histology (data not shown). Correlation between the macroscopic and pathological responses is summarized in Table 6, which indicates no correlation between them.
In the present study, 2 patients with CRs were observed. One was a 77 year-old man who had a small Borrman type II advanced gastric cancer at the pyloric ring, and biopsy demonstrated a poorly differentiated adenocarcinoma. After administration of UFT at $300 \mathrm{mg} /$ day for 23 days, endoscopic examination revealed complete disappearance of the ulcerative lesion, and the objective response was evaluated as CR. No ulcerative lesion was found macroscopically in the resected specimen; however, postoperative pathology demonstrated cancer cells infiltrating to the muscularis propria, and the histological response was evaluated as grade $1 \mathrm{~b}$. The postoperative stage classification was stage 1b: pT2 (pm), pN0, M0. He died of liver metastasis 46 months after the surgery. The second patient with CR was a 70 year-old women (Fig. 1), who had a large IIc-like advanced gastric cancer, and biopsy 
a1
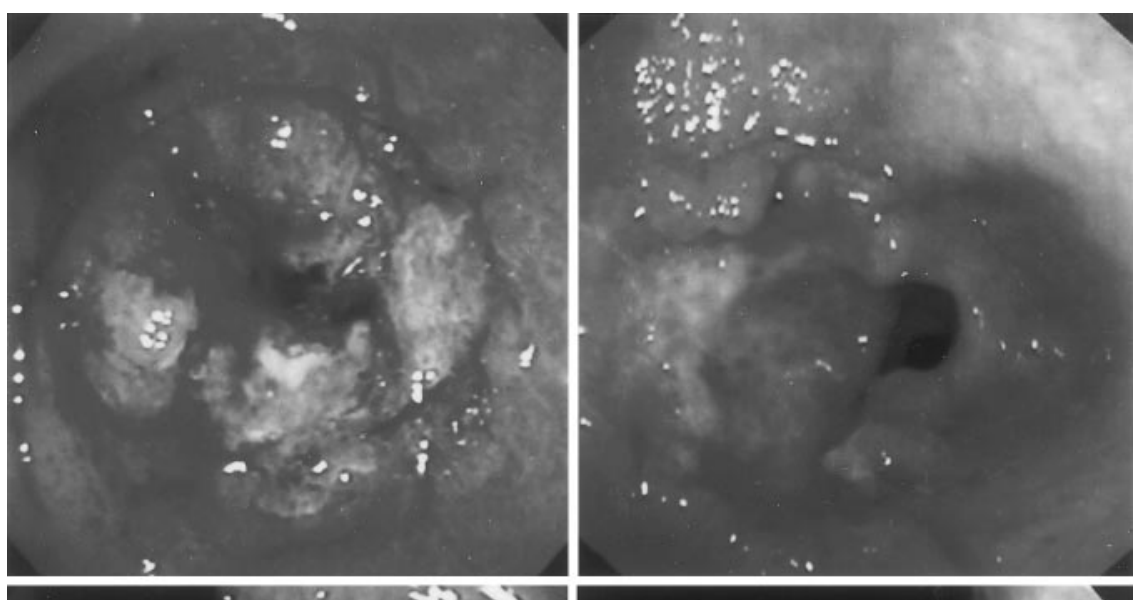

a2

b1
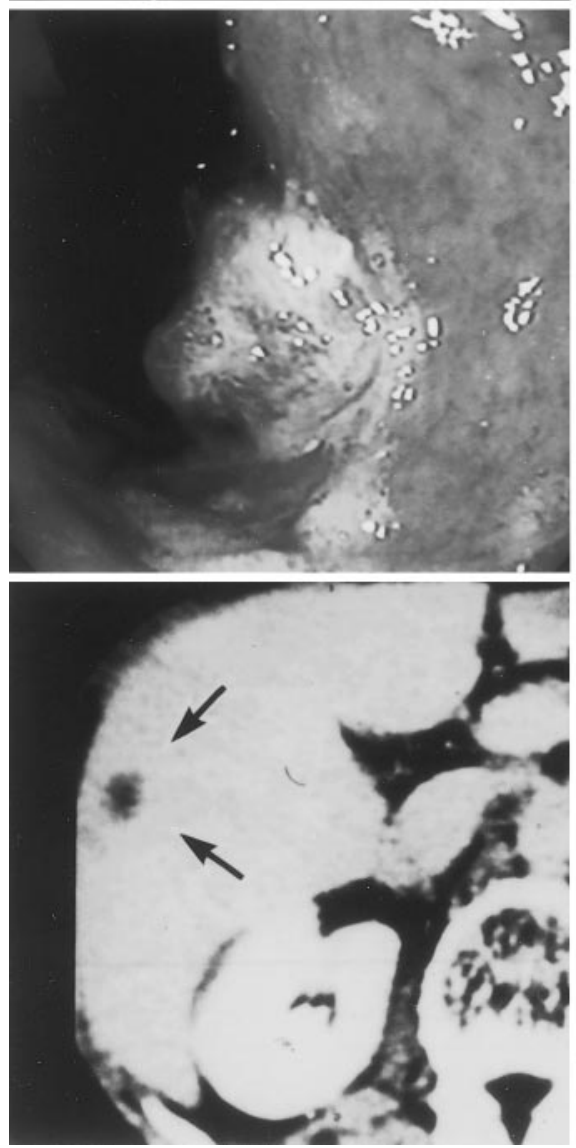
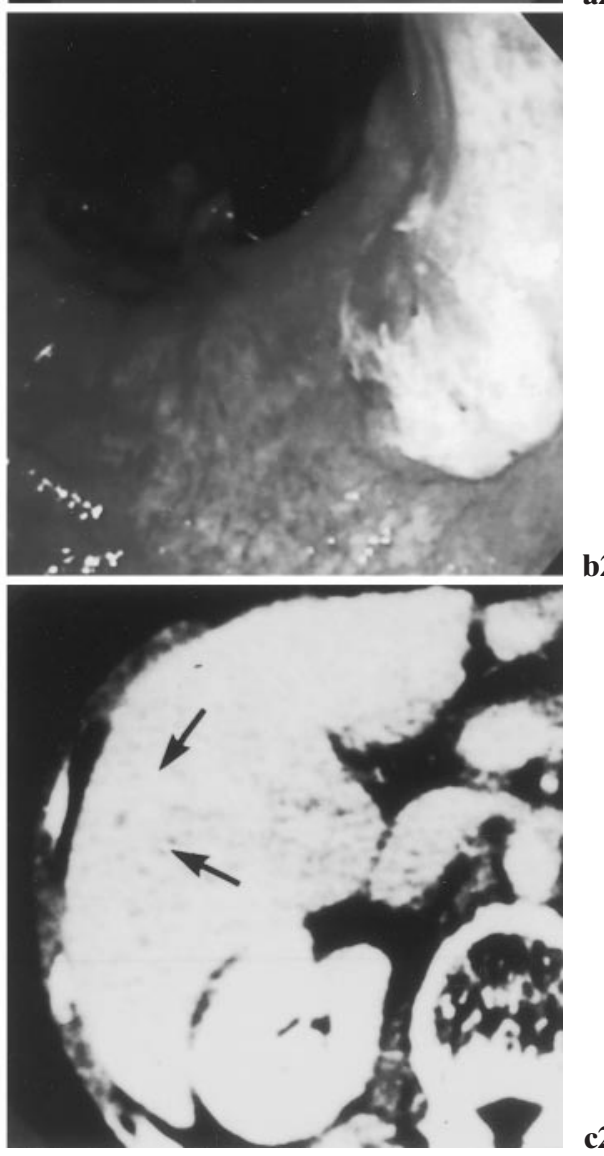

b2

Fig. 2a-c. Representative patients responding to treatment. a A 66-yearold woman diagnosed as Bor III (complete pyloric stenosis). a1 Before and $\mathbf{a} 2$ after UFT $300 \mathrm{mg} /$ day for 12 days. There was a partial response (size reduction) but stenosis was alleviated. b A 79-year-old man diagnosed as Bor I. b1 Before and b2 after UFT $300 \mathrm{mg} /$ day for 25 days. There was a partial response (size reduction). c A 50-year-old man diagnosed with liver metastasis. $\mathbf{c 1}$ Before and $\mathbf{c 2}$ after UFT $600 \mathrm{mg} /$ day for 35 days. There was a partial response in the primary lesion but the liver metastasis disappeared (arrows)
Table 6. Correlation between macroscopic and pathological responses

\begin{tabular}{lrrrrrr}
\hline & & \multicolumn{5}{c}{$\begin{array}{c}\text { Grade of pathological } \\
\text { response }\end{array}$} \\
\cline { 3 - 7 } Objective response & Total & 3 & 2 & $1 \mathrm{~b}$ & $1 \mathrm{a}$ & 0 \\
\hline CR & 2 & 0 & 1 & 1 & 0 & 0 \\
PR & 25 & 2 & 4 & 2 & 7 & 10 \\
NC & 42 & 0 & 4 & 6 & 14 & 18 \\
PD & 0 & 0 & 0 & 0 & 0 & 0 \\
Unevaluable & 13 & 0 & 2 & 2 & 6 & 3 \\
Total & 82 & 2 & 11 & 11 & 27 & 31 \\
\hline
\end{tabular}

demonstrated well-differentiated adenocarcinoma. After administration of UFT at $300 \mathrm{mg} /$ day for 16 days, endoscopic examination demonstrated the scar of the ulcerative lesion, and the objective response was evaluated as $\mathrm{CR}$, and no ulcerative lesion was found macroscopically. At first, the pathologists diagnosed no cancer cells remaining in the resected specimens; however, in a joint meeting of surgeons and pathologists, a minimal lesion was discovered in the submucosal layer, and the histological response was evaluated as grade 2. Her postoperative stage classification was stage 1a: pT1 (m), pN0, M0. Six years have passed since the surgery and 
Table 7. Drug concentration in tissues (29 patients)

\begin{tabular}{|c|c|c|c|}
\hline Dose (/day) & $600 \mathrm{mg}(n=6)$ & $400 \mathrm{mg}(n=13)$ & $300 \mathrm{mg}(n=10)$ \\
\hline Duration (days) & $21.7 \pm 10.1$ & $18.8 \pm 5.6$ & $20.2 \pm 8.5$ \\
\hline \multicolumn{4}{|l|}{ Primary lesion } \\
\hline FT $\mu \mathrm{g} / \mathrm{g}$ tissue & $3.928 \pm 3.329(n=5)$ & $1.567 \pm 1.719(n=9)$ & $1.168 \pm 0.721(n=5)$ \\
\hline 5-FU $\mu \mathrm{g} / \mathrm{g}$ tissue & $0.063 \pm 0.029(n=5)$ & $0.070 \pm 0.064(n=9)$ & $0.041 \pm 0.044(n=6)$ \\
\hline \multicolumn{4}{|l|}{ Normal mucosa } \\
\hline FT & $3.287 \pm 3.489(n=6)$ & $1.186 \pm 1.205(n=11)$ & $0.914 \pm 0.845(n=8)$ \\
\hline $5-\mathrm{FU}$ & $0.039 \pm 0.024(n=6)$ & $0.031 \pm 0.025(n=11)$ & $0.011 \pm 0.015(n=8)$ \\
\hline \multicolumn{4}{|l|}{ Metastatic node } \\
\hline FT & $1.478 \pm 2.688(n=3)$ & $3.624 \pm 2.700(n=4)$ & $0.0(n=2)$ \\
\hline $5-\mathrm{FU}$ & $0.069 \pm 0.072(n=3)$ & $0.041 \pm 0.050(n=4)$ & $0.0(n=2)$ \\
\hline \multicolumn{4}{|l|}{ Normal node } \\
\hline FT & $1.670 \pm 2.893(n=3)$ & $1.061 \pm 1.314(n=12)$ & $0.525 \pm 0.734(n=8)$ \\
\hline $5-\mathrm{FU}$ & $0.034 \pm 0.059(n=3)$ & $0.030 \pm 0.037(n=12)$ & $0.011 \pm 0.030(n=8)$ \\
\hline
\end{tabular}

FT, Futrafur; 5-FU, 5-fluorouracil

Table 8. Comparison of survival rates after surgery in patients who received neoadjuvant UFT and historical controls

\begin{tabular}{lll}
\hline & \multicolumn{2}{c}{ 3-year survival rates } \\
\cline { 2 - 3 } pTNM stage & \multicolumn{1}{c}{ Control $^{\mathrm{a}}$} & Neoadjuvant UFT \\
\hline Stage 1 & $94.2 \%(n=306)$ & $97.1 \%(n=40)$ \\
Stage 2 & $78.6 \%(n=38)$ & $75.0 \%(n=6)$ \\
Stage 3 & $61.3 \%(n=78)$ & $86.7 \%(n=19)$ \\
Stage 4 & $15.1 \%(n=117)$ & $41.6 \%(n=17)$ \\
\hline
\end{tabular}

${ }^{a}$ Historical controls in our department, 1980-1995

she is now hospitalized for rheumatoid arthritis, with no signs of recurrence.

\section{Drug concentration in tumor tissue (Table 7)}

All patients were given UFT at 6:00 p.m. on the day before surgery, and underwent surgery from 9:00 a.m. Blood was drawn at 9:00 a.m. and the tissues were taken between 11:00 a.m. and 3:00 p.m. in the operating room. Table 7 shows the drug concentrations in the cancerous and normal tissues, indicating that larger doses may be associated with a higher concentration of FT in the tissues. However, there were only slight differences in 5-FU concentrations among the three dosage groups.

\section{Survival after surgery}

All the patients underwent gastrectomy, and the survival curves after gastrectomy are shown in Fig. 3. During the follow-up period of 12-72 months (median period, 41 months), 16 patients died: 12 due to recurrence of the cancer and 4 of other diseases, including cerebral apoplexy, diabetes mellitus, sepsis caused by pneumonia, and myelodysplasia. Comparative survival rates for patients in this series at our department, and

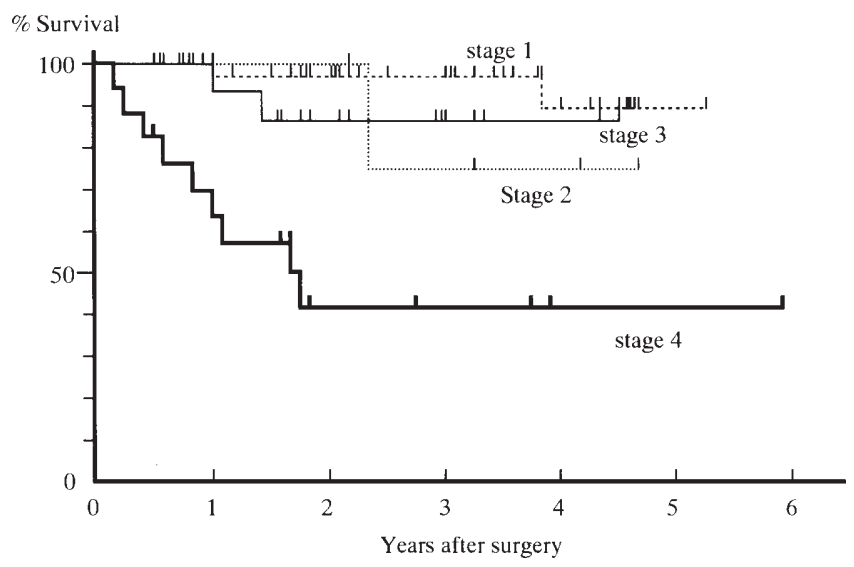

Fig. 3. Postoperative survival curves of patients who received neoadjuvant UFT. During the median follow-up period of 41 months, the 3-year survival rates were $97.1 \%$ for patients in stage $1(n=40), 75 \%$ for those in stage $2(n=6), 86.7 \%$ for those in stage $3(n=19)$, and $41.6 \%$ for those in stage $4(n=$ 17)

historical controls at the Japan Cancer Institute Hospital were calculated. The 3-year survival rates were $97.1 \%$ for stage $1(n=40), 75 \%$ for stage $2(n=6)$, $86.7 \%$ for stage $3(n=19)$, and $41.6 \%$ for stage $4(n=$ 17). These survival rates were higher than those of the historical controls in our department (3-year survival rates: $94 \%$ for stage 1,78\% for stage 2, $61 \%$ for stage 3 and $15 \%$ for stage 4 ) (Table 8 ).

The correlation between postoperative survival and the objective response is summarized in Fig. 4, indicating no difference in the survival curves between the $\mathrm{NC}$ group and the CR or PR groups.

\section{Discussion}

Since the reports of Wilke and colleagues [5], neoadjuvant chemotherapy of gastric cancer has 


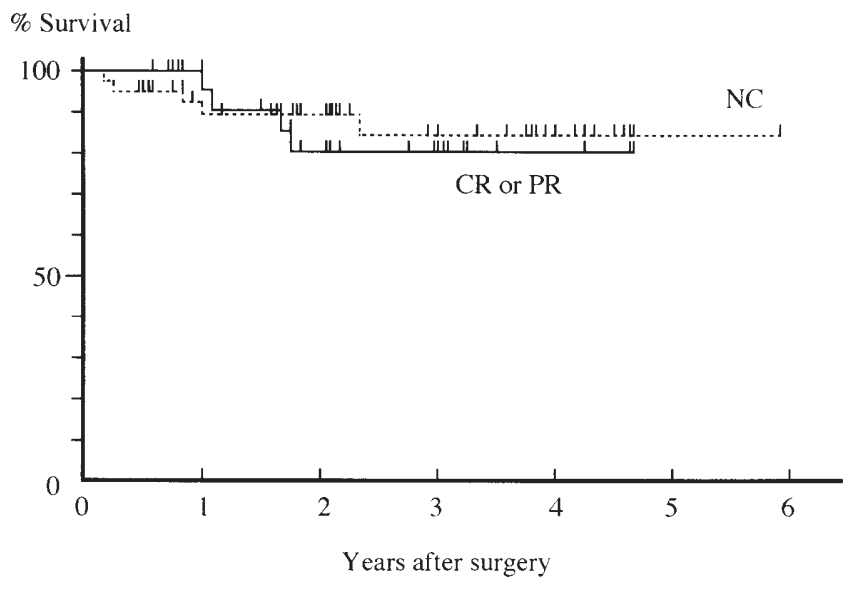

Fig. 4. Objective responses and postoperative survival curves after neoadjuvant UFT. Dotted lines, No change $(N C)(n=$ $42)$; continuous lines, CR or partial response $(P R)(n=27)$

attracted the interest of clinical oncologists, and neoadjuvant chemotherapy has been employed for the treatment of various malignancies, such as esophageal, breast, and gastric cancers. The major aim of neoadjuvant chemotherapy is downstaging for resectable cancer and improving the resectability of currently inoperable cancer. In the present study, neoadjuvant chemotherapy with UFT for gastric cancer achieved an objective response of $39 \%$, which is comparable to the responses produced by intensive chemotherapies, and compatible with previous reports on the clinical effects of oral UFT [23]. UFT is widely used for chemotherapy of digestive organ cancers in Japan, and response rates of various cancers to oral UFT are reported to be comparable to those of intensive i.v. chemotherapies, including CDDP, which usually achieve a $20 \%-40 \%$ response rate for gastric cancer. In addition, the side effects of UFT are not so severe [23]. Recently, it has also been reported that a combination regimen with UFT and leucovorin achieved a high response rate in colorectal cancer and was well tolerated [26,27]. These results suggest that UFT may be employed for neoadjuvant chemotherapy instead of intensive i.v. chemotherapy. In the present study, endoscopic examination showed that advanced gastric cancer became similar to early gastric cancer in several patients, and CT examination demonstrated the obvious shrinkage of metastatic nodes and disappearance of liver metastasis in two patients. These responses do not always suggest a preoperative downstaging, but preoperative downsizing of the primary and metastatic lesions was achieved in several patients by UFT.

It is unclear whether neoadjuvant therapy contributes to improved survival after surgery. There have been no reports on this subject, because it is difficult to define the effect of neoadjuvant therapy in the phase II setting. Six years have passed since the start of the present study, but the median follow-up duration is only 41 months. As shown in Table 8 , the survival rates of stage 3 and 4 patients were higher than those of the historical controls in our department, although the number of the patients was not sufficient for conclusive results to be drawn. Furthermore, comparisons with historical controls can be severely flawed. In addition, the present study demonstrated that there was no correlation between the objective response and the postoperative survival. Accordingly, if neoadjuvant UFT is truly benefical to improve survival, the macroscopic downsizing of primary tumors may not play an important role. In our previous report, an analysis using Akaike's information criteria demonstrated that the $\mathrm{N}$-factor as well as the T-factor were significant factors affecting the postoperative survival of gastric cancer patients [28]. Accordingly, one possible mechanism for the benefical effect of neoadjuvant chemotherapy on survival may be mediated by its effect on nodal involvment. Since the present study was not a randomized controlled study, such a trial with a larger sample size is necessary to clarify the true effects of neoadjuvant chemotherapy with UFT for gastric cancer.

Oral UFT also has much milder side effects than intensive i.v. chemotherapies. Previous reports showed that $41.4 \%$ of patients receiving oral UFT experienced side effects, but that the major side effects were anorexia $(24.3 \%)$, nausea and vomiting $(12.5 \%)$, and diarrhea $(11.1 \%)$; hematological toxicity was noted in only $6.9 \%$ of patients [23]. These results suggest an advantage of oral UFT in that patients can receive ambulatory chemotherapy without serious side effects. By contrast, intensive chemotherapy usually requires hospital care, due to its serious side effects, resulting in an impaired quality of life (QOL) and high cost. Accordingly, oral UFT may contribute to improving a patient's QOL and may also reduce treatment costs, making in much more suitable for neoadjuvant chemotherapy of gastric cancer than other intensive i.v. chemotherapies. However, in the present study, three patients became infected with MRSA and seven had moderate liver dysfunction after surgery. While all of these patients recovered, these complications seemed to be associated with the long period of administration ( $>4$ weeks). Accordingly, it is recommended that the dose of oral UFT should be reduced after 2 weeks of administration. Because the frequency of liver dysfunction with UFT therapy is reported to be $0.6 \%$ [23], the incidence of postoperative liver dysfunction in the present study seems to be high. The mechanisms responsible for postoperative liver dysfunction are unclear. Fluoropyrimidines, in general, sometimes cause serious liver dysfunction, and various medications after surgery, including drugs used for anesthesia and antibiotics in 
the present study, may work synergistically to cause liver dysfunction.

In the present study, UFT was administered at three different doses. Because UFT is an oral agent and one capsule includes $100 \mathrm{mg}$ of FT or one package includes $150 \mathrm{mg}$ of granules, it is difficult to administer a certain does per $\mathrm{kg}$ or per $\mathrm{m}^{2}$, and an obvious dose-dependency has not been seen [23]. The drug concentrations in the tissues may reflect this situation, because the FT concentration in cancer tissue was correlated with the dose administered, but the 5-FU concentration was not (Table 7). The reason for the lack of obvious dosedependency in chemotherapy with UFT is not clear. After oral administration, UFT may be partly degraded by stomach acid, absorbed by the gut, metabolized into 5-FU by the liver, and then accumulate in tumor tissues. Accordingly, differences among patients in stomach acid activity, gut absorption rates, and metabolizing enzyme activity in the liver may be involved. While no dose-dependent effects were noted in the present study, a higher response rate was seen in patients who received UFT for more than 3 weeks (Table 5). This result seems reasonable because the effect of 5-FU is timedependent. The results also suggests that $400 \mathrm{mg} /$ body for 3 weeks may be enough to achieve an objective response. In addition, in the present study, postoperative complications, especially MRSA enteritis and suture insufficiency, were seen in patients who received UFT at 400 or $600 \mathrm{mg} /$ body for more than 4 weeks. Accordingly, we recommend neoadjuvant chemotherapy with UFT at $400-600 \mathrm{mg} /$ body for the first 2 weeks and then at $300 \mathrm{mg} /$ body in order to reduce side effects and achieve a high response rate.

In conclusion, the present study indicates that preoperative chemotherapy for gastric cancer with oral UFT may result in clinical downsizing of primary tumors, as well as the prevention of tumor growth before surgery. Furthermore, preoperative chemotherapy with oral UFT is easy and safe, and patients can receive this treatment on an outpatient basis.

Acknowledgments. We gratefully acknowledge Ms. Tomoko Toga, Ms. Miyuki Ishihara, Ms. Yasuko Sonoyama, and Ms. Yuka Maniwa for their excellent secretarial assistance.

\section{References}

1. Wagener DJT, Yap SH, Wobbes T, Burghouts JTM, van Dam FE, Hillen HFP, et al. Phase II trial of 5-fluorouracil, adriamycin and cisplatin (FAP) in advanced gastric cancer. Cancer Chemother Pharmacol 1985;15:86-7.

2. Moertel CG, Rubin J, O'Connel MJ, Schutt AJ, Wieand HS. A phase II trial of 5-fluorouracil, doxorubicin, and cisplatin in the treatment of advanced upper gastrointestinal adenocarcinomas. J Clin Oncol 1986;4:1053-7.
3. Preusser P, Wilke H, Achterrath W, Fink U, Lenaz L, Heinicke A, et al. Phase II study with the combination etoposide, doxorubicin, and cisplatin in advanced measurable gastric cancer. J Clin Oncol 1989;7:1310-7.

4. Sparano JA, Schwartz EL, Salva KM, Pizzilo MF, Wadler S, Wiernik PH. Phase II trial of etoposide, doxorubicin (adriamycin) and cisplatin (EAP regimen) in advanced gastric cancer. Am J Clin Oncol (CCT) 1990;13:374-8.

5. Wilke H, Preusser U, Fink U, Gunzer U, Meyer HJ, Meyer J, et al. Preoperative chemotherapy in locally advanced and nonresectable gastric cancer: a phase II study study with etoposide, doxorubicin, and cisplatin. J Clin Oncol 1989;7:1318-26.

6. Stephens FO, Adams BG, Crea P. Intra-arterial chemotherapy given preoperatively in the management of carcinoma of the stomach. Surg Gynecol Obstet 1986;162:370-4.

7. Yonemura Y, Sawa T, Kinoshita K, Matsuki N, Fushida S, Tanaka S, et al. Neoadjuvant chemotherapy for high-grade advanced gastric cancer. World J Surg 1993;17:256-62.

8. Tanaka S, Ono M, Kobayashi J, Sakakibara N, Kameyama H. Application of Thio-TEPA before radical operation for gastric cancer. Jpn J Cancer Clin 1960;6:251-5.

9. Jinnai D, Tanaka S, Shimizu J, Ono M, Okajima K, Sakakibara N, et al. Histologic changes in the metastatic lymph nodes of stomach cancer after the preoperative application of anti cancer agent mitomycin C. Jpn J Cancer Clin 1962;8:533-43.

10. Fujimoto S, Watanabe Y, Enomoto K, Adachi M, Itoh K, Watanuki S. Studies on preoperative cancer chemotherapy: methods of preoperative intra-arterial infusion by the use of methotrexate or vinblastine. Cancer 1969;24:433-41.

11. Tani T, Shimura K, Nishimawari K, Koh T, Oride M, Niimoto M, et al. Clinical study on the oral administration of 5-fluorouracil tablet. Jpn J Cancer Chemother 1979;6:567-74.

12. Kawaura Y, Ohmura K, Hashizume Y, Takayama K, Sakatoku M, Hirano M, et al. Preoperative treatment with 5-fluorouracil (5FU) in gastric and colorectal cancer. Jpn J Cancer Chemother 1985;12:348-52.

13. Fujimoto S, Miyazaki M, Endoh F, Shimura T, Sugasawa H, Takahashi O, et al. Preoperative cancer chemotherapy for gastric cancer. Jpn J Cancer Chemother 1985;12:1010-8.

14. Kato M, Kinoshita K, Sawa T, Yoshimitsu S, Tomita F, Takano Y, et al. Neoadjuvant chemotherapy for far advanced gastric cancer. Jpn J Cancer Chemother 1990;17:PART-I, 391-6.

15. Ishihara $S$, Nakajima $T$, Ohta $K$, Tsuchiya S, Okumura S, Mizuno $\mathrm{S}$, et al. Evaluation of effective neo-adjuvant chemotherapy (FLEP therapy) in the treatment of advanced gastric cancer. Jpn J Cancer Chemother 1991;18:1748-52.

16. Chung Y-S, Kubo T, Onda N, Maeda K, Arimoto Y, Nitta A, et al. Neoadjuvant chemotherapy with EAP (etoposide, adriamycin, csiplatin) protocol for advanced gastric cancer - pilot study for histological effect and adaptation. J Jpn Soc Cancer Ther 1992;27:2064-71.

17. Ohmori Y, Kajitani T, Akiyama H, Abe O, Kusama S, Sakakibara $\mathrm{N}$, et al. Study of pre- and post-operative chemotherapy with oral 5-FU in patients with gastric cancer. Jpn J Cancer Chemother 1993;20;1781-9.

18. Kyoto Research Group for Chemotherapy of Gastric Cancer (KRGCGC): Tobe T, Nio Y, Tseng CC, Shiraishi T, Tsubono M, Kawabata K, et al. A randomized, comparative study of combination chemotherapies in advanced gastric cancers: 5-fluorouracil and cisplatin (FP) versus 5-fluorouracil, cisplatin, and 4'epirubicin (FPEPIR). Anticancer Res 1992;12:1983-8.

19. Nio Y, Tseng CC, Shiraishi T, Tsubono M, Kawabata K, Masai Y, et al. A phase II study of 5-fluorouracil, cisplatin and 4'-epirubicin (FPEPIR) in the treatment of advanced solid cancers. Am J Clin Oncol 1993;16:96-101.

20. Arima S, Ohsato K, Hisatsugu T, Shimura H. Multicenter randomized study of adjuvant chemotherapy with mitomycin-C and tegafur or tegafur-uracil in gastric cancer. Eur $\mathbf{J}$ Surg 1994:160:227-32. 
21. The Study group of Adjuvant Chemotherapy for Lung Cancer (Chubu, Japan). A randomized trial of postoperative adjuvant chemotherapy in non-small cell lung cancer. Eur J Surg Oncol 1995;21:69-77.

22. Ikeda K, Saitoh S, Koide I, Saitoh S, Koida I, Tsubota A, et al. A prospective randomized evaluation of a compound of tegafur and uracil as an adjuvant chemotherapy for hepatocellular carcinoma treated with transcatheter arterial chemoembolization. Am J Clin Oncol (CCT) 1995;18:204-10.

23. Ota K, Taguchi T, Kimura K. Report on nationwide pooled data and cohort investigation in UFT phase II study. Cancer Chemother Pharmacol 1988;22:333-8.

24. Miller AB, Hoogstraten B, Staquet M, Winkler A. Reporting results of cancer treatment. Cancer 1981;47:207-14.

25. Marunaka T, Umeno Y, Yoshida K, Nagamachi M, Minami Y, Fujii S. High pressure liquid chromatographic determination of futrafur [1-(tetrahydro-2-furanyl)-5-fluorouracil] and GLC-mass spectrometric determination of 5-fluorouracil and uracil in biological materials after oral administration of uracil plus futrafur. $\mathbf{J}$ Pharmacokinetic Sci 1980;69:1296-300.

26. Pazdur R, Lassere Y, Rhodes V, Ajani JA, Sugarman SM, Patt $\mathrm{YZ}$, et al. Phase II trial of uracil and tegafur plus oral leucovorin: an effective oral regimen in the treatment of metastatic colorectal carcinoma. J Clin Oncol 1994;12:2296-300.

27. Saltz LB, Leichman C, Young CW, Muggia FM, Conti JA, Spiess $\mathrm{T}$, et al. A fixed-ratio combination of uracil and futrafur (UFT) with low dose leucovorin. Cancer 1995;75:782-5.

28. Nio Y, Tsubono M, Kawabata K, Masai Y, Hayashi H, Meyer C, et al. Comparison of survival curves of gastric cancer patients after surgery according to the UICC stage classification and the general rules for gastric cancer study by the Japanese Research Society for Gastric Cancer. Ann Surg 1993;218:47-53. 